\title{
Le taux d'actualisation en France
}

\author{
par Bernard Walliser*
}

Le taux d'actualisation connaît un cheminement long et complexe entre sa définition théorique et son utilisation pratique.

\section{A. Historique}

Le taux d'actualisation est traditionnellement fixé par le Commissariat Général du Plan, en cohérence avec les objectifs macroéconomiques de la planification, soit en valeur réelle:

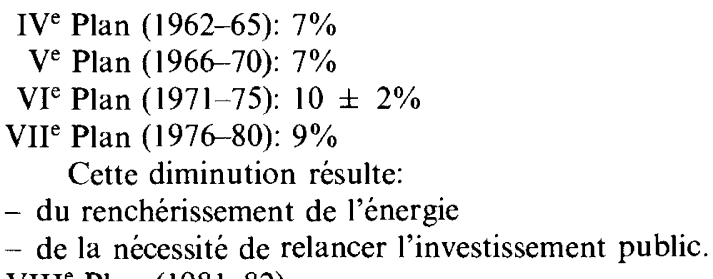

VIII ${ }^{\mathrm{e}}$ Plan (1981-82)

et Plan intérimaire (1982-83): 7\% (proposition non entérinée d'un groupe de travail de 1980)

Cette diminution fait suite à:

- un nouveau relèvement du prix de l'énergie

- une chute de la productivité apparente du capital et de sa rémunération $(-2$ à $-4 \%$ selon les hypothèses de déclassement).

IX ${ }^{\mathrm{e}}$ Plan (1984-88): 6\% (proposition non entérinée d'un groupe de travail de 1983-1984).

Cette diminution est due à une croissance ralentie et à la baisse poursuivie de la productivité apparente du capital.

$8 \%$ a été retenu en 1985, après négociation entre le Commissaire au Plan (qui souhaitait un faible taux d'actualisation) et le Ministre des Finances (qui optait pour un taux d'actualisation élevé), ce dernier mettant en avant:

- un risque croissant;

- une faible substitution capital-travail;

- des contraintes de financement.

* Professeur à l'Ecole Nationale des Ponts et Chaussées de Paris. 
Il résulte de ce qui précède que le taux d'actualisation a évolué sensiblement suivant une courbe en cloche. La comparaison du taux d'actualisation au taux d'intérêt réel révèle un écart en niveau de 6 à $10 \%$ et des variations respectives très déconnectées (le taux d'intérêt réel à long terme a crû légèrement au cours des dix dernières années).

\section{B. Méthodes de calcul}

Le taux d'actualisation est fondé sur la théorie micro-économique normative et représente un taux d'intérêt fictif (au sens de «shadow price»), nécessité par l'imperfection des marchés financiers dont le taux d'intérêt n'est donc pas un indicateur fiable de la rareté du capital.

1. Le taux d'actualisation se définit comme le rapport des prix d'un numéraire à 2 périodes consécutives:

- il est introduit théoriquement au niveau micro-économique où il sert de paramètre de décentralisation entre les décisions financières et techniques de l'entreprise;

- il est appréhendé pratiquement au niveau macro-économique en postulant son unicité (allocation efficace des investissements entre secteurs d'activité).

2. Le taux d'actualisation se détermine par un principe d'optimum inverse à partir d'un sentier de croissance retenu par les autorités politiques et traduisant l'arbitrage effectué entre consommation et investissement futurs. Ce taux est tel que, s'il était utilisé par tous les agents dans leur calcul de bilan actualisé, il conduirait à un volume d'investissement identique à celui du sentier de référence retenu. Deux approches restent possibles:

- la première, se fondant sur une fonction d'utilité collective, a été rejetée, cette fonction étant difficile à cerner;

- la seconde, s'appuyant sur une fonction de production agrégée, a été retenue, et conduit à un taux d'actualisation égal à la productivité marginale nette du capital, sur le sentier de croissance privilégié.

3. Un calcul direct du taux d'actualisation en niveau, sur le secteur industriel uniquement (car la croissance de ce secteur n'est pas trop heurtée, contrairement aux autres secteurs), a été effectué (voir annexe):

- à l'aide d'une fonction de production de type clay-clay (c'est-à-dire à générations de capital et d'emploi, sans substitution entre facteurs ni ex ante ni ex post);

- à partir de deux scénarios à l'horizon 2000, définis par le modèle macro-économique simplifié «mini-DMS-long terme» (DMS = dynamique multisectorielle), et prolongeant les projections à l'horizon 1988 du modèle de base DMS.

4. Un calcul indirect du taux d'actualisation est possible, en variation par rapport à une période de référence (1968-73) de croissance régulière $(6 \%)$ où il est considéré comme bien défini (10\%); il suffit de mesurer l'impact de modifications des conditions exogènes en s'appuyant soit sur la liaison théorique entre taux d'actualisation et taux de croissance, soit sur la liaison entre taux d'actualisation et productivité apparente du capital (voir annexe). 


\section{Problèmes méthodologiques associés}

Le taux d'actualisation est principalement un paramètre de décentralisation global pour l'économie, d'autres considérations devant être prises en compte à travers d'autres paramètres plutôt qu'internalisées dans un concept unique.

1. En ce qui concerne l'incertitude, il convient de tenir compte des risques collectifs (progrès technique, environnement international) par une augmentation du taux d'actualisation. Par contre, les risques spécifiques au projet ne doivent pas être pris en compte, car ils s'annulent en moyenne au niveau global, l'Etat jouant le rôle d'assureur de ces petits risques pour les projets publics.

2. Le taux d'actualisation est défini sur l'ensemble de l'économie et traduit les contraintes financières globales. Mais il existe des contraintes financières spécifiques au secteur public en raison de la rareté des ressources publiques. Au lieu de jouer sur le taux d'actualisation, on a introduit un coût d'opportunité des fonds publics appliqué seulement aux dépenses budgétaires par un coefficient de majoration (1,3 à 1,5 dans le passé).

3. Le taux d'actualisation est aussi lié aux déséquilibres macro-économiques (surtout le déficit de la balance commerciale et le sous-emploi). Des simulations ont montré que la variation du taux d'actualisation était sans effet sur ces deux déséquilibres; sa méthode de calcul reste donc valable, sinon sa valeur.

Mais pour résorber les déséquilibres, on peut jouer sur les deux autres des trois prix fictifs associés aux ressources primaires de l'économie, à savoir:

- un taux d'intérêt fictif pour le capital;

- un taux de change fictif pour les biens importés;

- un salaire fictif pour le travail.

Un groupe de travail du Plan est arrivé à la conclusion qu'il était impossible de fixer un salaire fictif, mais possible de fixer un prix fictif pour les biens importés, pénalisant ceux-ci de 20 ou $30 \%$ par un protectionnisme indirect.

4. Les règles de choix d'investissement et de tarification doivent être compatibles, la tarification marginale de long terme s'appuyant sur le taux d'actualisation.

\section{Modalités d'utilisation}

Le taux d'actualisation est mis en œuvre dans des créneaux de décision bien particuliers quant aux entreprises concernées et aux types de projets.

1. Le calcul économique classique est utilisé essentiellement par les entreprises publiques de monopole (plutôt que concurrentielles) dans leurs choix internes et les négociations avec leur tutelle. Il fait l'objet de méthodologies standard à EDF (Electricité de France) et à la Direction des Routes, et donne lieu à des études plus ponctuelles à la SNCF (chemin de fer) et aux Télécommunications. 


\section{Annexe - Le modèle de calcul}

\section{Données:}

$$
\begin{array}{ll}
\delta_{\mathrm{t}} & =\text { coefficient d'actualisation } \\
\mathrm{Q}_{\mathrm{t}} & =\text { production } \\
\mathrm{p}_{\mathrm{t}} & =\text { prix de la production } \\
\mathrm{I}_{\mathrm{t}} & =\text { investissement } \\
\mathrm{r}_{\mathrm{t}} & =\text { coût d'usage du capital } \\
\mathrm{N}_{\mathrm{t}} & =\text { emploi } \\
\mathrm{w}_{\mathrm{t}} & =\text { salaire horaire } \\
\mathrm{a} & =\text { taux d'actualisation } \\
\mu & =\text { taux de déclassement } \\
\mathrm{g} & =\text { taux de croissance } \\
\mathrm{S} & =\text { taux d'épargne } \\
\alpha & =\text { rémunération des facteurs } \\
\pi & =\text { productivité apparente du capital }
\end{array}
$$

Méthode directe:

$$
\begin{array}{lll}
\max _{\mathrm{I}_{\mathrm{t}}} \sum_{\mathrm{t}=0}^{\infty} \delta_{\mathrm{t}}\left(\mathrm{p}_{\mathrm{t}} \mathrm{Q}_{\mathrm{t}}-\mathrm{r}_{\mathrm{t}} \mathrm{I}_{\mathrm{t}}-\mathrm{w}_{\mathrm{t}} \mathrm{N}_{\mathrm{t}}\right) & \text { profit futur actualisé } \\
\mathrm{I}_{\mathrm{t}} \quad \mathrm{t}=0 & \\
\mathrm{~K}_{\mathrm{t}} \quad=\mathrm{g}_{\mathrm{t}}\left(\mathrm{I}_{\mathrm{t}}, \ldots, \mathrm{I}_{\mathrm{v}}, \ldots\right) & \\
\mathrm{N}_{\mathrm{t}}=\mathrm{h}_{\mathrm{t}}\left(\mathrm{I}_{\mathrm{t}} \ldots, \mathrm{I}_{\mathrm{v}}, \ldots\right) & \text { fonction de production clay-clay } \\
\mathrm{Q}_{\mathrm{t}}=\mathrm{f}_{\mathrm{t}}\left(\mathrm{K}_{\mathrm{t}}, \mathrm{N}_{\mathrm{t}}\right) &
\end{array}
$$

donne une chronique $\delta_{\mathrm{t}}=\frac{\delta \mathrm{t}-1}{1+\mathrm{a}_{\mathrm{t}}}$ tel que $\mathrm{r}_{\mathrm{v}} \cdot \delta_{\mathrm{v}}=\sum_{\mathrm{t}=\mathrm{v}+1}^{\infty} \delta_{\mathrm{t}} \quad \mathrm{p}_{\mathrm{t}} \frac{\delta \mathrm{f}_{\mathrm{t}}}{\delta \mathrm{K}_{\mathrm{t}}} \cdot \frac{\delta \mathrm{g}_{\mathrm{t}}}{\delta \mathrm{I}_{\mathrm{v}}}-\mathrm{w}_{\mathrm{t}} \frac{\delta \mathrm{N}_{\mathrm{t}}}{\delta \mathrm{I}_{\mathrm{v}}}$

Si la fonction de production est de type Cobb-Doublas:

$$
\mathrm{a}_{\mathrm{v}}=\frac{\delta \mathrm{fv}}{\delta \mathrm{K}}-\mu
$$

Méthode indirecte:

- Liaison taux d'actualisation-taux de croissance: $a=\pi-\mu$

- Liaison taux d'actualisation-productivité apparente du capital:

$$
\mathrm{a}=(\lambda-a) \cdot \frac{\mathrm{g}+\mu}{\mathrm{s}}-\mu
$$


2. Pour une gamme de projets dont le taux de rentabilité avait été calculé, on a pu constater la décision finalement retenue. Seules quelques interversions par rapport au calcul ont été observées (projets peu rentables acceptés, projets rentables refusés). En revanche, peu de comparaisons entre la rentabilité ex ante et la rentabilité ex post ont été effectuées, du fait de la difficulté à appréhender cette dernière.

3. Le taux d'actualisation n'a de sens que si tous les agents l'utilisent; tel n'est pas le cas, même dans le secteur public, de sorte que la concurrence est faussée entre Electricité de France et Gaz de France, de même qu'entre les chemins de fer et les transporteurs routiers (dans ce cas, le taux d'actualisation de 6\% adopté par la SNCF est cependant proche du niveau de rentabilité du secteur routier).

4. Une distorsion entre le bilan économique et le bilan financier d'une entreprise apparaît du fait de l'utilisation de prix fictifs; un problème analogue se pose d'ailleurs lorsqu'un bilan intègre des effets non marchands (valeur de la vie humaine, valeur du temps). La possibilité de compensation de cet écart par la tutelle soulève alors un problème de révélation de l'information pertinente dont dispose l'entreprise, problème encore mal résolu. 